COMMUNICATIONS IN

ANALYSIS AND GEOMETRY

Volume 11, Number 5, 987-999, 2003

\title{
Weil-Petersson Translation Distance and Volumes of Mapping Tori
}

\author{
JEFFREY F. BROCK
}

Given a closed hyperbolic 3-manifold $T_{\psi}$ that fibers over the circle with monodromy $\psi: S \rightarrow S$, the monodromy $\psi$ determines an isometry of Teichmüller space with its Weil-Petersson metric whose translation distance $\|\psi\|_{\text {WP }}$ is positive. We show there is a constant $K \geqslant 1$ depending only on the topology of $S$ so that the volume of $T_{\psi}$ satisfies

$$
\|\psi\|_{\mathrm{WP}} / K \leqslant \operatorname{vol}\left(T_{\psi}\right) \leqslant K\|\psi\|_{\mathrm{wP}}
$$

\section{Introduction.}

In this paper we generalize our study of the relation of Weil-Petersson distance to volumes of hyperbolic 3-manifolds to the context of closed hyperbolic 3-manifolds that fiber over $S^{1}$.

Let $S$ be a closed surface of genus at least 2, and let $\operatorname{Mod}(S)$ denote its mapping class group, or the group of isotopy classes of orientation preserving self-homeomorphisms of $S$. Let $\psi$ be a pseudo-Anosov element of $\operatorname{Mod}(S)$, i.e. an element $\psi$ so that no positive power of $\psi$ preserves any isotopy class of simple closed curves on the surface other than the trivial one.

Elements of $\operatorname{Mod}(S)$ act by isometries on the Teichmüller space Teich $(S)$ with its Weil-Petersson metric, which arises naturally from an $L^{2}$ inner product on the cotangent space to $\operatorname{Teich}(S)$ at $X$ weighted by the hyperbolic metric on $X$. Letting $d_{\mathrm{WP}}(.,$.$) denote Weil-Petersson distance, let$

$$
\|\psi\|_{\mathrm{WP}}=\inf _{X \in \operatorname{Teich}(S)} d_{\mathrm{WP}}(X, \psi(X))
$$

denote the Weil-Petersson translation distance of $\psi$ acting as an isometry of $\operatorname{Teich}(S)$.

Choosing a particular representative $\hat{\psi}: S \rightarrow S$ of $\psi$, we may form the mapping torus

$$
T_{\psi}=S \times[0,1] /(x, 1) \sim(\hat{\psi}(x), 0)
$$

\footnotetext{
${ }^{1}$ Research partially supported by NSF grant DMS-9971721.
} 
whose topology depends only on $\psi$. Thurston exhibited a hyperbolic structure on $T_{\psi}$ for any pseudo-Anosov $\psi$; the hyperbolic volume $\operatorname{vol}\left(T_{\psi}\right)$ is then naturally associated to the mapping class $\psi$. The question of whether the assignment $\psi \rightarrow \operatorname{vol}\left(T_{\psi}\right)$ is a previously encountered isotopy invariant of a homeomorphism is a natural one.

This paper relates the hyperbolic volume of $T_{\psi}$ to the Weil-Petersson translation distance for $\psi$.

Theorem 1.1. Given $S$ there is a constant $K>1$ and so that if $T_{\psi}$ is a pseudo-Anosov mapping torus, we have

$$
\frac{1}{K}\|\psi\|_{\mathrm{WP}} \leqslant \operatorname{vol}\left(T_{\psi}\right) \leqslant K\|\psi\|_{\mathrm{WP}}
$$

The proof of Theorem 1.1 mirrors the proof of a similar result for quasiFuchsian hyperbolic 3-manifolds [Br]: if $Q(X, Y)=\mathbb{H}^{3} / \Gamma(X, Y)$ is the hyperbolic quotient of $\mathbb{H}^{3}$ by the Bers simultaneous uniformization of the pair $(X, Y) \in \operatorname{Teich}(S) \times \operatorname{Teich}(S)$, then we have the following theorem.

Theorem 1.2 (Main Theorem of $[\mathrm{Br}]$ ). Given the surface $S$, there are constants $K_{1}>1$ and $K_{2}>0$ so that

$$
\frac{d_{\mathrm{WP}}(X, Y)}{K_{1}}-K_{2} \leqslant \operatorname{vol}(\operatorname{core}(Q(X, Y))) \leqslant K_{1} d_{\mathrm{WP}}(X, Y)+K_{2} .
$$

Here, core $(Q(X, Y))$ is the convex core of $Q(X, Y)$ : the smallest convex subset of $Q(X, Y)$ carrying its fundamental group.

The challenge of Theorem 1.1 is primarily to relate the techniques involved in the proof of Theorem 1.2 to the setting of 3-manifolds fibering over $S^{1}$. Here is a quick sketch of the argument:

- The cover $M_{\psi}$ of $T_{\psi}$ corresponding to the inclusion $\iota: S \rightarrow T_{\psi}$ of the fiber is an infinite volume doubly degenerate manifold homeomorphic to $S \times \mathbb{R}$ and invariant by a translational isometry $\Psi: M_{\psi} \rightarrow M_{\psi}$, for which $\Psi_{*}=\psi_{*}$.

- We control the volume of a fundamental domain $D$ for the action of $\Psi$ from below by estimating up to bounded error a lower bound for the number of bounded length closed geodesics in $D$. Since $D$ is not convex, there is potential spill-over of volume into other translates of $D$; this is rectified by a limiting argument. 
- We control the volume of $T_{\psi}$ from above by building a model manifold $N_{\Delta} \cong T_{\psi}$ built out of 3 -dimensional tetrahedra, and a degree-1 homotopy equivalence $f: N_{\Delta} \rightarrow T_{\psi}$ that is simplicial (the lift $\widetilde{f}: \widetilde{N_{\Delta}} \rightarrow \mathbb{H}^{3}$ sends each tetrahedron to the convex hull of the image of its vertices). A priori bounds on the volume of a tetrahedron in $\mathbb{H}^{3}$ give an estimate on the total volume of the image, and by a spinning trick (as in $[\mathrm{Br}]$ ) we may homotope $f$ to force all but $k\|\psi\|_{\text {WP }}$ to have arbitrarily small volume.

The constructions in each case are directly those of [Br], to which we refer often in the interest of brevity. The paper will introduce vocabulary in section 2 and prove the main theorem in section 3 .

Convergence of iteration to the fiber. The sequence of quasi-Fuchsian manifolds $Q\left(\psi^{-n}(X), \psi^{n}(Y)\right)$ simultaneously uniformizing the Riemann surfaces $\psi^{-n}(X)$ and $\psi^{n}(Y)$ at infinity converges strongly to $M_{\psi}$ (in both the algebraic and geometric topologies; see, e.g. [Th2] [Mc]). Another approach to Theorem 1.1 could compare the volumes of compact subsets of $Q_{n}=Q\left(\psi^{-n}(X), \psi^{n}(Y)\right)$ to their limits in $M_{\psi}$ using geometric convergence.

In particular, if one could show that the diameter of the convex core of $Q\left(\psi^{-n}(X), \psi^{n}(Y)\right)$ grows linearly with $n$, the inflexibility theory of McMullen in $[\mathrm{Mc}]$ could be used to show that

$$
\operatorname{vol}\left(\operatorname{core}\left(Q_{n}\right)\right)=n \operatorname{vol}\left(T_{\psi}\right)+O(1)
$$

which would give a proof of Theorem 1.1 that uses only the statement of Theorem 1.2 rather than elements of its proof. Such diameter estimates are undertaken in joint work of the author with K. Bromberg that generalizes McMullen's inflexibility theory to the setting without injectivity bounds $[\mathrm{BB}]$. We thank the referee for pointing out this alternate line of argument.

Acknowledgements. I would like to thank Yair Minsky and Dick Canary for inspirational discussions on the topic of this paper, and the referee for valuable observations.

\section{Vocabulary.}

We define our terms. Throughout, we assume for simplicity that $S$ is a closed surface of genus at least 2 .

Teichmüller space. The Teichmüller space Teich $(S)$ is the set of marked hyperbolic structures on $S$, namely pairs $(f, X)$ of a hyperbolic surface $X$ and 
a homeomorphism $f: S \rightarrow X$, up to the equivalence relation $(f, X) \sim(g, Y)$ if there is an isometry $\phi: X \rightarrow Y$ so that $\phi \circ f \simeq g$. The Teichmüller space inherits a topology from the marked bi-Lipschitz distance:

$$
d_{\mathrm{bL}}((f, X),(g, Y))=\inf _{\phi \simeq g \circ f^{-1}} \log (L(\phi))
$$

where

$$
L(\phi)=\sup _{x \in X, v \in T_{x}(X)}|D \phi(v)| /|v|
$$

denotes the best bi-Lipschitz constant for $\phi$.

Considering $X=\mathbb{H} / \Gamma$ as a Riemann surface, let $Q(X)$ denote the vector space of holomorphic quadratic differentials $\phi(z) d z^{2}$ on $X$. The space $Q(X)$ is naturally the holomorphic cotangent space to Teich $(S)$. The WeilPetersson Hermitian metric on Teich $(S)$ is induced by the inner product on $Q(X)$

$$
\langle\phi, \psi\rangle_{\mathrm{WP}}=\int_{X} \frac{\phi \bar{\psi}}{\rho^{2}} d z d \bar{z}
$$

(where $\rho(z)|d z|$ is the Poincaré metric on $X$ ) via the duality $(\mu, \phi)=\int_{X} \mu \phi$ where $\mu$, a Beltrami differential, is a tangent vector to Teich $(S)$. Here, we consider the Riemannian part $g_{\mathrm{WP}}$ of the Weil-Petersson metric, and its associated distance function $d_{\mathrm{WP}}(.,$.$) .$

We will often refer to a hyperbolic Riemann surface $X$ as an element of Teich $(S)$, assuming an implicit marking $(f: S \rightarrow X)$.

Hyperbolic 3-manifolds. Moving up a dimension, we denote by

$$
\mathcal{V}(S)=\operatorname{Hom}\left(\pi_{1}(S), \operatorname{Isom}^{+}\left(\mathbb{H}^{3}\right)\right) / \text { conjugation }
$$

the representation variety for $\pi_{1}(S)$. The subset $A H(S) \subset \mathcal{V}(S)$ represents the locus of equivalence classes $[\rho]$ for which $\rho$ is discrete and faithful. Each equivalence class $[\rho]$ lying in $A H(S)$ determines a complete hyperbolic 3manifold $M=\mathbb{H}^{3} / \rho\left(\pi_{1}(S)\right)$. Since hyperbolic 3-manifolds are $K(\pi, 1) \mathrm{s}$, points in $A H(S)$ are in bijection with the set of all marked hyperbolic 3manifolds $(f: S \rightarrow M)$, where $f$ is a homotopy equivalence, modulo the equivalence relation $(f: S \rightarrow M) \sim(g: S \rightarrow N)$ if there is an isometry $\phi: M \rightarrow N$ so that $\phi \circ f \simeq g$.

Simple closed curves. Let $\mathcal{S}$ denote the set of isotopy classes of essential simple closed curves on $S$. Given two curves $\alpha$ and $\beta$ in $\mathcal{S}$ their geometric intersection number $i(\alpha, \beta)$ is obtained by counting the minimal number of points of intersection over all representatives of $\alpha$ and $\beta$ on $S$. 
A pants decomposition $P$ of $S$ is a maximal collection of distinct elements of $\mathcal{S}$ with pairwise disjoint representatives on $S$. Two pants decompositions $P$ and $P^{\prime}$ are related by an elementary move if $P^{\prime}$ can be obtained from $P$ by removing a curve $\alpha \in P$ and replacing it with a curve $\beta \neq \alpha$ so that $i(\alpha, \beta)$ is minimal among all choices for $\beta$ (see figure 1 ). Denote by $\mathbf{P}(S)$ the pants graph whose vertices are pants decompositions of $S$ and whose edges join pants decompositions differing by an elementary move. Hatcher

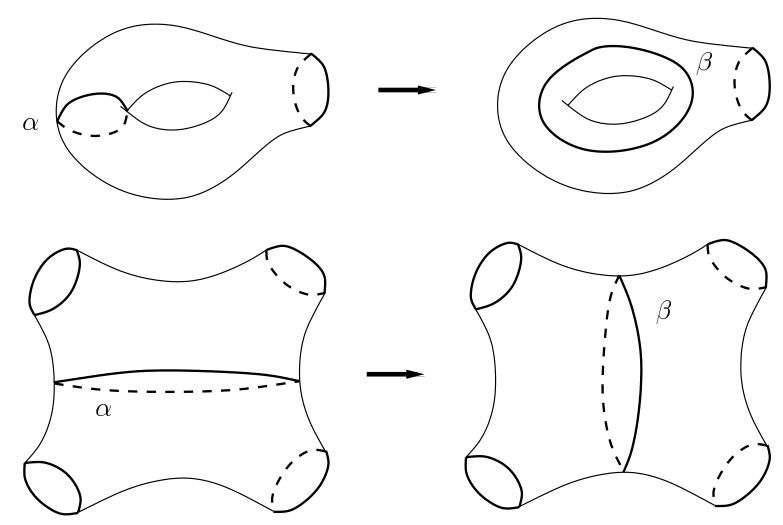

Figure 1: Elementary moves on pants decompositions.

and Thurston introduced the graph $\mathbf{P}(S)$ in [HT], where they proved $\mathbf{P}(S)$ is connected (see also [HLS]). It therefore carries a natural path metric, obtained by assigning each edge length 1 . We will be interested in the distance between vertices: we let $d_{\mathbf{P}}\left(P, P^{\prime}\right)$ to be the length of a minimal length path in $\mathbf{P}(S)$ joining $P$ to $P^{\prime}$.

In $[\mathrm{Br}]$ we proved the following theorem.

Theorem 2.1. The graph $\mathbf{P}(S)$ is coarsely quasi-isometric to $\operatorname{Teich}(S)$ with its Weil-Petersson metric.

(See [Br, Thm 1.1]). A quasi-isometry $Q: \mathbf{P}(S) \rightarrow$ Teich $(S)$ is obtained by taking $Q$ to be any mapping for which $Q(P)=X_{P}$ lies in the set

$$
V(P)=\left\{X \in \operatorname{Teich}(S) \mid \ell_{X}(\alpha)<L \text { for each } \alpha \in P\right\}
$$

where $L$ is Bers's constant, namely, the minimal constant for which each hyperbolic surface $X \in \operatorname{Teich}(S)$ admits a pants decomposition $P$ so that each $\alpha \in P$ has geodesic representative with length less than $L$ on $X$ (see [Bus]). 
To avoid cumbersome notation, we will view $\mathbf{P}(S)$ as a collection of pants decompositions equipped with a distance $d_{\mathbf{P}}(.,$.$) and refer to a pants$ decomposition $P$ as an element of $\mathbf{P}(S)$ rather than making specific reference to $P$ as a vertex of $\mathbf{P}(S)$.

Simplicial hyperbolic surfaces. Let $\operatorname{Sing}_{k}(S)$ denote the marked singular hyperbolic structures on $S$ with at most $k$ cone singularities, namely surfaces that are hyperbolic away from at most $k$ cone points, each with cone angle at least $2 \pi$, equipped with homeomorphisms $g: S \rightarrow Z$, up to marking preserving isometry. As with Teich $(S), \operatorname{Sing}_{k}(S)$ is topologized via the marked bi-Lipschitz distance, defined analogously.

Let $M$ be a complete hyperbolic 3-manifold. A simplicial hyperbolic surface is a path-isometric map $h: Z \rightarrow M$ of a singular hyperbolic surface into $M$ so that $h$ is totally geodesic on the faces of a geodesic triangulation $T$ of $Z$, in the sense of [Hat] (cf. [Br, Sec. 4]). We say $g$ has associated triangulation $T$. If $T$ is a one-vertex triangulation, and $\alpha$ is a closed loop represented by an edge of $T$ that maps to the closed geodesic reprentative $h(\alpha)^{*}$ of $h(\alpha)$ in $M$, we say $h$ is adapted to $\alpha$, following Canary.

Given $M=(f: S \rightarrow M)$ in $A H(S)$, we denote by $\mathcal{S H}_{k}(M)$ the simplicial hyperbolic surfaces homotopic to $f$, in other words, those simplicial hyperbolic surfaces $h: Z \rightarrow M$, so that if $g: S \rightarrow Z$ is the marking on $Z$, then $h \circ g$ is homotopic to $f$.

As in $[\mathrm{Br}]$, the following result of Canary (see [Can, Sec. 5]) will be instrumental in what follows.

Theorem 2.2 (Canary). Let $M \in A H(S)$ have no cusps, and let $\left(g_{1}, Z_{1}\right)$ and $\left(g_{2}, Z_{2}\right)$ lie in $\mathcal{S} \mathcal{H}_{1}(M)$ where $\left(g_{1}, Z_{1}\right)$ and $\left(g_{2}, Z_{2}\right)$ are adapted to simple closed curves $\alpha$ and $\beta$ on $S$. Then there is a continuous family $\left(g_{t}: Z_{t} \rightarrow\right.$ $M) \in \mathcal{S H}_{2}(M), t \in[1,2]$.

\section{Proof of the main theorem.}

Given $\psi \in \operatorname{Mod}(S)$, let $T_{\psi}$ be the mapping torus for $\psi$. By a theorem of Thurston ([Th2], see also $[\mathrm{Mc}],[\mathrm{Ot}]), T_{\psi}$ admits a complete hyperbolic structure if and only if $\psi$ is pseudo-Anosov: no power of $\psi$ fixes any isotopy class of essential simple closed curves on $S$.

To prove Theorem 1.1 we relate the volume of the mapping torus $T_{\psi}$ to the translation distance of $\psi$ on the pants graph $\mathbf{P}(S)$.

Proof: (of Theorem 1.1) Let $M_{\psi}$ be the cover of $T_{\psi}$ corresponding to the inclusion of the fiber $\iota: S \rightarrow T_{\psi}$; $\iota$ naturally lifts to a marking $\left(\tilde{\iota}: S \rightarrow M_{\psi}\right)$ 
of $M_{\psi}$, so $M_{\psi}$ is naturally an element of $A H(S)$.

We will apply the techniques of [Br] directly to larger and larger subsets of the manifold $M_{\psi}$.

Bounding volume from below. Consider any simplicial hyperbolic surface $\left(h: Z \rightarrow M_{\psi}\right) \in \mathcal{S H}_{1}\left(M_{\psi}\right)$. Let $\left(h_{\psi}: Z_{\psi} \rightarrow M_{\psi}\right) \in \mathcal{S H}_{1}\left(M_{\psi}\right)$ be the simplicial hyperbolic surface obtained by post-composing the image with the generating covering transformation $\Psi: M_{\psi} \rightarrow M_{\psi}$ acting on $\pi_{1}\left(M_{\psi}\right)$ by $\psi_{*}$.

The hyperbolic surface $Z^{\mathrm{h}}$ in the same conformal class as $Z$ differs from the hyperbolic surface $Z_{\psi}^{\mathrm{h}}$ by the action of $\psi$ on Teich $(S)$. If $Z^{\mathrm{h}}$ lies in $V(P)$, then, the surface $Z_{\psi}^{\mathrm{h}}$ lies in $V(\psi(P))$.

By an argument using Theorem 2.2 in an exactly analogous manner to its use in [Br, Sec. 4], there is a sequence

$$
g_{\psi}=\left\{P=P_{0}, P_{1}, \ldots, P_{n}=\psi(P)\right\}
$$

and a continuous family $\left(h_{t}: Z_{t} \rightarrow M_{\psi}\right) \subset \mathcal{S H}_{k}\left(M_{\psi}\right), k=2,0 \leqslant t \leqslant n$, of simplicial hyperbolic surfaces joining $Z$ and $Z_{\psi}$ so that the following holds:

- For each $i$, the simplicial hyperbolic surface $h_{i}: Z_{i} \rightarrow M_{\psi}$ satisfies $\ell_{Z_{i}^{\mathrm{h}}}(\alpha)<L$ for each $\alpha \in P_{i}$.

- Successive pants decompositions have distance $d_{\mathbf{P}}\left(P_{i}, P_{i+1}\right)<k$ where $k$ depends only on $S$.

Given a set $X$, let $\mathcal{P}(X)$ denote its power set, or its set of subsets. Denote by $\pi_{\mathcal{S}}: \mathcal{P}(\mathbf{P}(S)) \rightarrow \mathcal{P}(\mathcal{S})$ mapping that assigns to each collection of pants decompositions in $\mathbf{P}(S)$ the union of all curves they comprise.

We may then apply the following lemma of [Br].

Lemma 3.1 (Lemma 4.2 of $[\mathrm{Br}]$ ). Let $k \in \mathbb{N}$ and let

$$
g=\left\{P_{I}=P_{0}, \ldots, P_{N}=P_{T}\right\} \subset \mathbf{P}(S)
$$

be a sequence of pants decompositions with the property that $d_{\mathbf{P}}\left(P_{j}, P_{j+1}\right)<$ $k$. Let $\mathcal{S}_{g} \subset \mathcal{S}$ denote the image of $g$ under the projection $\pi_{\mathcal{S}}$. There is a constant $K_{0}>0$ depending on $k$ and $S$ so that

$$
d_{\mathbf{P}}\left(P_{I}, P_{T}\right)<K_{0}\left|\mathcal{S}_{g}\right|
$$


Let

$$
g_{j}=\left\{g_{\psi}, \psi\left(g_{\psi}\right), \ldots, \psi^{j}\left(g_{\psi}\right)\right\}
$$

be a sequence of pants decompositions resulting from the concatenation of the sequences $\psi^{i}\left(g_{\psi}\right)=\left\{\psi^{i}\left(P_{0}\right), \ldots, \psi^{i}\left(P_{n}\right)\right\}$ for $i=0, \ldots, j$. Noting that $\psi^{i}\left(P_{n}\right)=\psi^{i+1}\left(P_{0}\right)$, the sequence of pants decompositions $g_{j}$ satisfies the hypotheses of Lemma 3.1 so we have

$$
d_{\mathbf{P}}\left(P, \psi^{j}(P)\right) \leqslant K_{0}\left|\mathcal{S}_{g_{j}}\right| .
$$

Consider the image of the homotopy $H:[0, n] \times S \rightarrow M_{\psi}$ for which $H(t,)=.h_{t} \circ g_{t}: S \rightarrow M_{\psi}$ and $g_{t}: S \rightarrow Z_{t}$ is the implicit marking on $Z_{t}$.

The image $H([0, n] \times S)$ is a compact subset of $M_{\psi}$; choose an embed$\operatorname{ding} h: S \rightarrow M_{\psi}$ homotopic to $\iota$ so that $h(S)$ does not intersect the unit neighborhood $\mathcal{N}_{1}(H([0, n] \times S))$ of the image $H([0, n] \times S)$.

Let

$$
\Psi: M_{\psi} \rightarrow M_{\psi}
$$

be the isometric covering transformation so that

$$
\iota \circ \psi \simeq \Psi \circ \iota
$$

Let $n_{0}$ be an integer so that $\Psi^{n_{0}} \circ h(S)$ is also disjoint from $\mathcal{N}_{1}(H([0, n] \times S))$ and so that $\mathcal{N}_{1}(H([0, n] \times S))$ lies in the compact subset $\mathcal{K}$ of $M_{\psi}$ bounded by $h(S)$ and $\Psi^{n_{0}} \circ h(S)$. Note that

$$
\operatorname{vol}\left(\mathcal{K}_{n_{0}}\right)=n_{0} \operatorname{vol}\left(T_{\psi}\right)
$$

since the region bounded by each $\Psi^{i} \circ h(S)$ and $\Psi^{i+1} \circ h(S)$ is a fundamental domain for the action on $\Psi$ on $M_{\psi}$. It follows that if we set

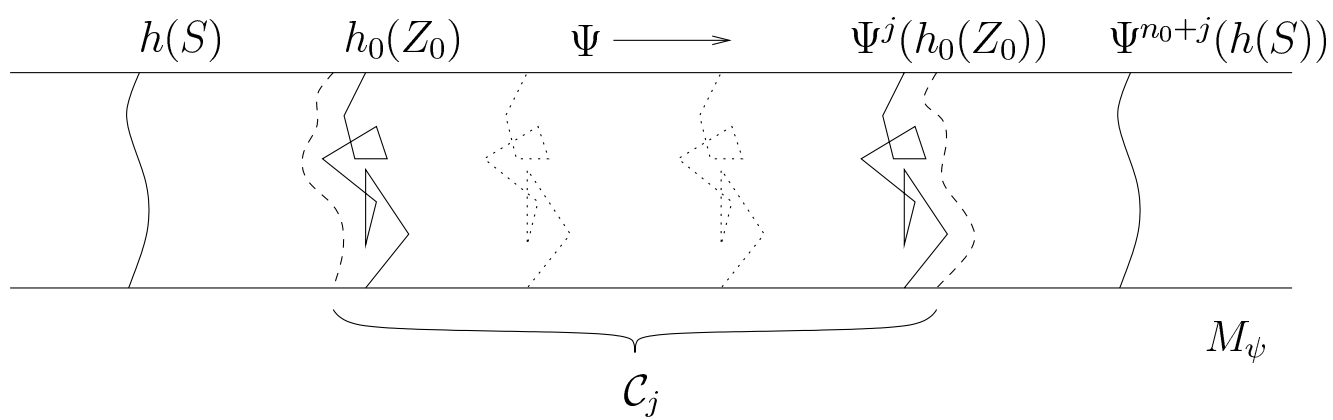

Figure 2: Bounding volume from below. 


$$
\mathcal{C}_{j}=\bigcup_{i=0}^{j} \Psi^{i}\left(\mathcal{N}_{1}(H([0, n] \times S))\right)
$$

then we have

$$
\left(n_{0}+j\right) \operatorname{vol}\left(T_{\psi}\right) \geqslant \operatorname{vol}\left(\mathcal{C}_{j}\right) .
$$

The subset $\mathcal{C}_{j}$ has the property that for each $\alpha \in \mathcal{S}_{g_{j}}$ the unit neighborhood of the geodesic representative $\alpha^{*}$ of $\alpha$ lies in $\mathcal{C}_{j}$. Applying the argument of Lemma 4.1 of [Br], there are constants $c_{1}>1$ and $c_{2}>0$ depending only on $S$ so that $\mathcal{C}_{j}$ has volume

$$
\operatorname{vol}\left(\mathcal{C}_{j}\right)>\frac{\left|\mathcal{S}_{g_{j}}\right|}{c_{1}}-c_{2} \geqslant \frac{d_{\mathbf{P}}\left(P, \psi^{j}(P)\right)}{c_{1} K_{0}}-c_{2} .
$$

Applying Theorem 2.1, we have constants $d_{1}>1$ and $d_{2}>0$ so that

$$
d_{\mathbf{P}}\left(P, \psi^{j}(P)\right) \geqslant \frac{d_{\mathrm{WP}}\left(Z, \psi^{j}(Z)\right)}{d_{1}}-d_{2} \geqslant \frac{j\|\psi\|_{\mathrm{WP}}}{d_{1}}-d_{2}
$$

where $\|\psi\|_{\text {WP }}$ is the Weil-Petersson translation distance of $\psi$.

Thus there are $k_{1}>1$ and $k_{2}>0$ so that we have

$$
\left(n_{0}+j\right) \operatorname{vol}\left(T_{\psi}\right) \geqslant \frac{j\|\psi\|_{\mathrm{WP}}}{k_{1}}-k_{2}
$$

Taking the limit as $j$ tends to infinity, we have

$$
\operatorname{vol}\left(T_{\psi}\right) \geqslant \frac{\|\psi\|_{\mathrm{WP}}}{k_{1}}
$$

Bounding volume from above. To bound $\operatorname{vol}\left(T_{\psi}\right)$ from above, we adapt our construction of a simplicial model 3-manifold for a quasi-Fuchsian 3-manifold in [Br, Sec. 5] to build a triangulated 3-manifold $N \cong T_{\psi}$, and a degree one homotopy equivalence $f: N \rightarrow T_{\psi}$ that is simplicial: the lift $\widetilde{f}: \widetilde{N} \rightarrow \mathbb{H}^{3}$ maps each simplex $\Delta \subset N$ to the convex hull of the images of its vertices.

Consider the surface $Z^{\mathrm{h}}$ in $V(P)$, and let $T$ be a standard triangulation suited to $P$, in the sense of [Br, Defn. 5.3]. Let $\left(g_{0}: Z_{0} \rightarrow M_{\psi}\right) \in \mathcal{S H}_{k}\left(M_{\psi}\right)$ be a simplicial hyperbolic surface with associated triangulation $T$ that realizes each $\alpha \in P$ : the geodesic representative of $\alpha$ in $Z_{0}$ is mapped to its geodesic representative in $M$. As in [Br] we may choose $\left(g_{0}: Z_{0} \rightarrow M_{\psi}\right)$ so that the vertices of $T$ map to pairs of antipodal vertices on each geodesic $\alpha^{*}$. In other words, the two vertices that lie in $\alpha^{*}$ separate $\alpha^{*}$ into two segments of the same length. 
Let $\left(g_{1}: Z_{1} \rightarrow M_{\psi}\right)$ be the simplicial hyperbolic surface

$$
g_{1}=\Psi \circ g_{0} \circ \psi^{-1} .
$$

Since $g_{1} \circ \psi$ is homotopic to $\Psi \circ g_{0}$, the simplicial hyperbolic surface $\left(g_{1}: Z_{1} \rightarrow\right.$ $\left.M_{\psi}\right)$ lies in $\mathcal{S H}_{k}\left(M_{\psi}\right)$. Moreover, $g_{1}$ realizes the pants decomposition $\psi(P)$ and is adapted to $\psi(T)$.

As shown in $[\mathrm{Br}]$, there is a triangulated model 3 -manifold $N \cong S \times I$ built out of tetrahedra, together with a simplicial mapping $g: N \rightarrow M_{\psi}$ with the following properties:

1. There is a constant $k$ so that all but $k d_{\mathbf{P}}(P, \psi(P))$ of the tetrahedra in $N$ have the property that one edge maps by $g$ to a geodesic $\alpha^{*}$, where $\alpha \in \mathcal{S}_{g}$.

2. If $\partial^{-} N=S \times\{0\}$ and $\partial^{+} N=S \times\{1\}$, then $\left.g\right|_{\partial^{-} N}$ factors through the simplicial hyperbolic surface $\left(g_{0}: Z_{0} \rightarrow M_{\psi}\right)$, and $\left.g\right|_{\partial^{+} N}$ factors through the simplicial hyperbolic surface $\left(g_{1}: Z_{1} \rightarrow M_{\psi}\right)$.

After adjusting $\left.g\right|_{\partial^{+} N}$ by precomposition with an isotopy, we may glue $\partial^{-} N$ to $\partial^{+} N$ by a homeomorphism $h \simeq \psi$ to obtain a manifold $N_{\psi} \cong T_{\psi}$, and a homotopy equivalence $f_{\psi}: N_{\psi} \rightarrow T_{\psi}$. By a degree argument the map $f_{\psi}$ is surjective.

There is a constant $\mathcal{V}_{3}$ so that the volume of each tetrahedron in $\mathbb{H}^{3}$ is less than $\mathcal{V}_{3}$ (see [Th1]), so the volume of the image of $g$ is less than $\mathcal{V}_{3}$ times the number of tetrahedra in $N$. Arguing as in [Br, Sec. 5], we may spin the mapping $f_{\psi}$ by pulling the vertices $p_{\alpha}$ and $\bar{p}_{\alpha}$ around the geodesic $\alpha^{*}$, keeping the map simplicial. In the process, the volume of the image of each tetrahedron $\Delta$ in $N$ for which $g$ sends an edge of $\Delta$ to $\alpha^{*}$ for some $\alpha \in \mathcal{S}_{g}$, can be made arbitrarily small by spinning the mapping sufficiently far (see [Br, Lem. 5.10]).

For each $\epsilon>0$, we may spin the mapping $f_{\psi}$ to a mapping $f_{\psi}^{\theta}$ so that the image of $f_{\psi}^{\theta}$ has volume less than

$$
k \mathcal{V}_{3} d_{\mathbf{P}}(P, \psi(P))+\epsilon .
$$

It follows from Theorem 2.1 that there are constants $e_{1}>1$ and $e_{2}>0$ so that

$$
\operatorname{vol}\left(T_{\psi}\right) \leqslant e_{1} d_{\mathrm{WP}}(Z, \psi(Z))+e_{2} .
$$

But there is a constant $C(Z)$ depending on the surface $Z$, so that

$$
d_{\mathrm{WP}}\left(Z, \psi^{n}(Z)\right) \leqslant C(Z)+\left\|\psi^{n}\right\|_{\mathrm{WP}}=C(Z)+n\|\psi\|_{\mathrm{WP}} .
$$


(The constant $C(Z)$ measures twice the distance to the invariant WeilPetersson geodesic in $\operatorname{Teich}(S)$ for the action of the isometry $\psi$. See [DW, Wol]).

Since $\operatorname{vol}\left(T_{\psi^{n}}\right)=n \operatorname{vol}\left(T_{\psi}\right)$, we have

$$
n \operatorname{vol}\left(T_{\psi}\right) \leqslant e_{1}\left(C(Z)+n\|\psi\|_{\mathrm{WP}}\right)+e_{2} .
$$

Taking limits of both sides as $n \rightarrow \infty$, we have

$$
\operatorname{vol}\left(T_{\psi}\right) \leqslant e_{1}\|\psi\|_{\mathrm{WP}}
$$

Setting $K=\max \left\{k_{1}, e_{1}\right\}$ proves the theorem.

Remark: I. Agol has recently given a sharp upper bound for volume in terms of the translation distance on $\mathbf{P}(S)$ [Ag, Cor. 2.4].

Computing translation distance. We conclude with a brief discussion of the computability of the Weil-Petersson translation distance. In particular, one might ask whether there is an effective or algorithmic way to compute the Weil-Petersson translation distance of a pseudo-Anosov transformation $\psi \in \operatorname{Mod}(S)$. The invariant Weil-Petersson geodesic for $\psi$ is obtained via a minimization process and as such admits no concrete parametrization.

It is possible that the combinatorial model $\mathbf{P}(S)$ for the Weil-Petersson metric might lend itself to a more algorithmic study. The theory of curve hierarchies developed by Masur and Minsky [MM1, MM2] produces a quasigeodesic path in $\mathbf{P}(S)$ by an inductive construction using geodesics in the complex of curves $\mathcal{C}(S)$. A recent Ph.D. thesis of J. Leasure gives an algorithm for finding geodesics joining given pairs of points in $\mathcal{C}(S)$ [Le]. Such an algorithm should suffice to give an algorithm to produce such a MasurMinsky curve hierarchy and thus an algorithmic method to join a pair of points in $\mathbf{P}(S)$ by a quasi-geodesic.

It would be interesting to seek a further algorithm to minimize the length of such hierarchies joining $P$ to $\psi(P)$ over pants decompositions $P \in \mathbf{P}(S)$, as this would give a means to compute Weil-Petersson translation distance of $\psi$ up to uniformly bounded additive and multiplicative error.

\section{References.}

[Ag] I. Agol. Small 3-manifolds of large genus. Preprint, arXiv:mathGT/0205091 (2002).

[Br] J. Brock. The Weil-Petersson metric and volumes of 3-dimensional hyperbolic convex cores. J. Amer. Math. Soc. 16 (2003), 495-535. 
[BB] J. Brock and K. Bromberg. Geometric inflexibility of hyperbolic 3-manifolds. In preparation (2002).

[Bus] P. Buser. Geometry and Spectra of Compact Riemann Surfaces. Birkhauser Boston, 1992.

[Can] R. D. Canary. A covering theorem for hyperbolic 3-manifolds and its applications. Topology 35 (1996), 751-778.

[DW] G. Daskolopoulos and R. Wentworth. Classification of WeilPetersson isometries. Amer. J. Math. 125 (2003), no. 4, 941-975.

[Hat] A. Hatcher. On triangulations of surfaces. Topology Appl. 40 (1991), 189-194.

[HLS] A. Hatcher, P. Lochak, and L. Schneps. On the Teichmüller tower of mapping class groups. J. Reine Angew. Math. 521 (2000), 1-24.

[HT] A. Hatcher and W. Thurston. A presentation for the mapping class group. Topology 19 (1980), 221-237.

[Le] J. Leasure. Geodesics in the Complex of Curves of a Surface. Ph.D. Thesis, U. Texas, Austin, 2002.

[MM1] H. Masur and Y. Minsky. Geometry of the complex of curves I: hyperbolicity. Invent. Math. 138 (1999), 103-149.

[MM2] H. Masur and Y. Minsky. Geometry of the complex of curves II: hierarchical structure. Geom. \& Funct. Anal. 10 (2000), 902-974.

[Mc] C. McMullen. Renormalization and 3-Manifolds Which Fiber Over the Circle. Annals of Math. Studies 142, Princeton University Press, 1996.

[Ot] J. P. Otal. Le théorème d'hyperbolisation pour les variétés fibrées de dimension trois. Astérisque, 1996.

[Th1] W. P. Thurston. Geometry and Topology of Three-Manifolds. Princeton lecture notes, 1979.

[Th2] W. P. Thurston. Hyperbolic structures on 3-manifolds II: Surface groups and 3-manifolds which fiber over the circle. Preprint, arXiv:math.GT/9801045 (1986). 
[Wol] S. Wolpert. The geometry of the Weil-Petersson completion of Teichmüller space. Preprint (2002).

Math Department, University of Chicago

5734 S. University Ave., Chicago, IL 60637

brock@math.uchicago.edu

ReCeived January 9, 2002. 